Editorial

\title{
Acknowledgement to Reviewers of Journal of Energy and Power Technology in 2021
}

Journal of Energy and Power Technology Editorial Office

LIDSEN Publishing Inc., 2000 Auburn Drive, One Chagrin Highlands, Suite 200, Beachwood, OH, USA; E-Mail: jept@lidsen.com

Journal of Energy and Power Technology

2022, volume 4, issue 1

doi:10.21926/jept.2201001
Received: January 05, 2022

Accepted: January 06, 2022

Published: January 06, 2022

\begin{abstract}
The editors of Journal of Energy and Power Technology would like to express their sincere gratitude to the following reviewers for assessing manuscripts in 2021. We greatly appreciate the contribution of expert reviewers, which is crucial to the journal's editorial process. We aim to recognize reviewer contributions through several mechanisms, of which the annual publication of reviewer names is one. Reviewers receive a voucher entitling them to a discount on their next LIDSEN publication and can download a certificate of recognition directly from our submission system. Additionally, reviewers can sign up to the service Publons (https://publons.com) to receive recognition. Of course, in these initiatives we are careful not to compromise reviewer confidentiality. Many reviewers see their work as a voluntary and often unseen part of their role as researchers. We are grateful to the time reviewers donate to our journals and the contribution they make.
\end{abstract}

The editors of Journal of Energy and Power Technology would like to express their sincere gratitude to the following reviewers for assessing manuscripts in 2021.

We greatly appreciate the contribution of expert reviewers, which is crucial to the journal's editorial process. We aim to recognize reviewer contributions through several mechanisms, of which the annual publication of reviewer names is one. Reviewers receive a voucher entitling them to a discount on their next LIDSEN publication and can download a certificate of recognition directly from our submission system. Additionally, reviewers can sign up to the service Publons

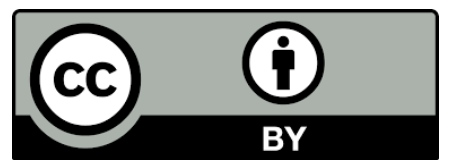

(C) 2022 by the author. This is an open access article distributed under the conditions of the Creative Commons by Attribution License, which permits unrestricted use, distribution, and reproduction in any medium or format, provided the original work is correctly cited. 
(https://publons.com) to receive recognition. Of course, in these initiatives we are careful not to compromise reviewer confidentiality. Many reviewers see their work as a voluntary and often unseen part of their role as researchers. We are grateful to the time reviewers donate to our journals and the contribution they make.

If you are interested in becoming a reviewer for Journal of Energy and Power Technology, see the link at the bottom of the webpage http://lidsen.com/joinus.

The following reviewed for Journal of Energy and Power Technology in 2021:

\begin{tabular}{|l|l|l|}
\hline Agbulut, Umit & Huang, Junjie & Ramezani Amin \\
\hline Aghajani, Hossein & Huang, Zongyu & Sánchez-Martínez, Araceli \\
\hline Ali, Muhammad & Ismail, Mohammad & Saraji, Soheil \\
\hline Almeida, C. Marisa R. & Kaldellis, John & Saxén, Henrik \\
\hline Amado, Miguel & Kamel Abo-Elyousr, Farag & Senthil, Ramalingam \\
\hline Avellaneda, David & Karami, Hamidreza R. & Sheng Jiang \\
\hline Aydoĝn, Şakir & Karamov, Dmitriy N. & Shu, Jie \\
\hline Baglivo, Cristina & Kerrouche, K. & Stauffer, Philip \\
\hline Balasubramanian, K. R. & Laptev, Alexander M. & Sudhakar, Kumarasamy \\
\hline Bennett, Gary L. & Leśniak, Magdalena & Sulowicz, Maciej \\
\hline Brunklaus Gunther & Li, Hong-Ru & Sun, Xuping \\
\hline Burkel Eberhard & Li, Meicheng & Syahputra, Ramadoni \\
\hline Cazarez-Candia, O. & Lin, Zhongwei & Tamura, Junji \\
\hline Cervone, Angelo & Liu, Gao & Tavares, Maria Cristina Dias \\
\hline Chen, Chong & Liu, Jinyun & Thapar, Sapan \\
\hline Chen, Zhen & Liu, Tao & Valencia, Felipe \\
\hline Cholewa, Tomasz & Liu, Wen & Velasquez, Carlos E. \\
\hline Chowdhury, Amor & Liu, Xuepeng & Wang Hongzhi \\
\hline Coelho, Pedro & Lorenc-Grabowska, Ewa & Wang, Li \\
\hline Cung, Khanh & Luttenberger, Lidija Runko & Watanabe, Hikari \\
\hline Dai, Haifeng & Lyubina, Julia & Wildfeuer, Leo \\
\hline Dan, Ho Kim & Martínez, Herminio & Wu, Rui \\
\hline Deng, Wenwen & Michalak, Piotr & Xavier, Lúcia Helena \\
\hline Ding, Chao & Minakshi, Manickam & Xiao, Jie \\
\hline Ding, Yu & Mlonka-Mędrala, Agata & Yan F. \\
\hline Elias, Said & Moghimi, M. A. & Yao Chunde \\
\hline Fan, Wei & Narula, Kapil & Ye Dingding \\
\hline Feng, Jinkui & Nezhad, Meysam Majidi & Ye, Hua \\
\hline Feng, Ju & Padoin, Natan & Ye, Tengling \\
\hline Feng, Xuning & Pakhuruddin, Mohd Zamir & Yuan, Bin \\
\hline Gabrielli, Fabrizio & Pan, Bin & Yuan, Qiusheng \\
\hline Garapati, Nagasree & Pan, Chunjian & Yuan, Tao \\
\hline Ghosh, A. & Zanadim \\
\hline Glowacz, Adam & Zhang, Jianbin \\
\hline Gopalan, Raghavan & \\
\hline
\end{tabular}




\begin{tabular}{|l|l|l|}
\hline Habibi, Hamed & Peña-Garcia, R. & Zhang, Kai \\
\hline Hassan, Qusay & Perne, Matija & Zhang, Liwei \\
\hline Holappa, Lauri & Piechota, Grzegorz & Zhang, Peng \\
\hline Hooper, Justin B. & Qiu, Yingning & Zhao, Kai \\
\hline Hu, Rong & & \\
\hline
\end{tabular}

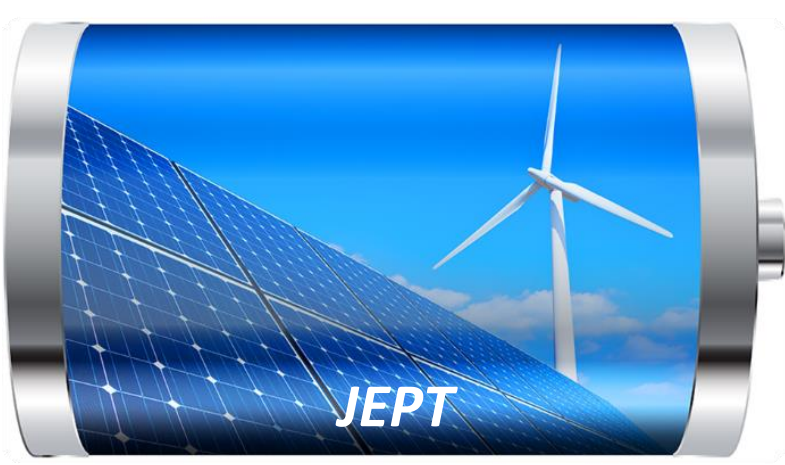

Enjoy JEPT by:

1. Submitting a manuscript

2. Joining in volunteer reviewer bank

3. Joining Editorial Board

4. Guest editing a special issue

For more details, please visit:

http://www.lidsen.com/journal/jept 